Z B Research Square

\title{
The Effects of Weapon Toys on Aggressive Behavior among Chinese Children: The Mediating Role of Aggressive Cognition
}

Qian Zhang ( 453457096@qq.com)

Southwest University

Research article

Keywords: weapon toys, aggressive cognition, aggressive behavior, children

Posted Date: August 10th, 2021

DOl: https://doi.org/10.21203/rs.3.rs-771437/v1

License: (9) (7) This work is licensed under a Creative Commons Attribution 4.0 International License. Read Full License 


\begin{abstract}
Background: The purpose of the study was to explore the relationships between exposure to weapon toys and aggressive behavior.

Methods: The study sample included 104 six-year-old kindergarten children. Half of them were randomly assigned to play with weapon toys (experimental group) or non-weapon toys (control group). A Semantic Classification Task (SCT) and a Competitive Reaction Time Task (CRTT) were employed to measure aggressive cognition and aggressive behavior. A randomized controlled study was performed and a 2 x 2 (Toys, Gender) Analysis of Covariance (ANCOVA) statistical test was used to evaluate the effect of toys on aggressive cognition (reaction times to weapon pictures) and behavior (noise intensity setting), controlling for age. Mediation analysis was also conducted to test the mediating role of aggressive cognition in this effect.
\end{abstract}

Results: Results indicated that playing with weapon toys increased aggressive cognition and aggressive behavior. Specifically, boys were more likely than girls to exhibit aggressive behavior in the weapon toys condition, though girls did exhibit aggressive cognition. Mediation analysis indicated that the weapon toy effect on aggressive behavior was partially mediated by aggressive cognition.

Conclusions: The main findings of the study suggest that boys may be considered as the target group for aggression intervention and reduction in the context of weapon toys. Practitioners can take away the weapon toys to reduce aggressive cognition, so as to decrease children's aggressive behavior.

\title{
Background
}

Aggression usually includes implicit aggression (e.g., cognitive aggression) and explicit aggression (e.g., behavioral aggression). Implicit aggression can be measured through hostile cognition and anger (Bushman \& Huesmann, 2010). Explicit aggression is defined as any behavior directed toward another individual that is carried out with the proximate or immediate intent to cause harm (Anderson \& Bushman, 2002). In addition, the perpetrator must believe that their behavior will harm the target who is motivated to avoid the behavior (Geen, 2001). Drawing upon this, we assume that aggression consists of aggressive cognition and aggressive behavior. Due to ethical reasons in this study, aggressive cognition is operationally conceptualized as a reaction time (RT) to weapon/aggressive pictures (e.g., guns, swords) in the SCT. Aggressive behavior is conceptualized as the noise intensity (0-4 points) children set for "virtual opponent" in Step 2 of the CRTT (a proxy external behavior).

\section{Theoretical Models of Aggression}

To date, the general aggression model (GAM) is a representative theory to explain the mechanism underlying the effect of media violence on aggression (Anderson \& Bushman, 2018). The GAM posits that situtational factor (e.g., game, movie, weapon) and personal factor (e.g., sex, age, trait) affect aggressive thoughts, aggressive cognition, hostile feeling and aggressive behavior (Bartholow et al., 2005). In addition, social cognitive theory (SCT) assumes that children mimic aggressive behavior from role models through cognition (Bandura, 1999). However, some scholars criticize these models and propose opposite theories (like the Catalyst Model) from an evolutionary perspective that explicitly suggest media has little impact (Surette \& Maze, 2015). Given that the GAM and the SCT explain the specific mechanisms from violent cues to aggressive cognition and to aggressive behavior, weapon toys (as a type of violent cues) may affect both aggressive cognition and behavior.

\section{Children and Weapon Toys}

Weapon toys (or toy weapons) refer to all kinds of weapon-shaped toys played by children, such as knives, swords, scissors, slingshots, guns, etc (Rosenblum, 2007). Nowadays, playing with weapon toys has been one of the the favorite leisure activities among 4-7-year-old children in China (see

https://www.jianshu.com/p/e9b1494186a0). It is necessary to examine the effect of weapon toys on social behavior in this particular age group. Thus we can help them to manage their weapon toys well and mitigate possible negative weapon toy effects on them. The past literature indicates that exposure to violent media increases aggressive thoughts, aggressive cognition, aggressive affect, physiological arousal, and aggressive behavior (Anderson \& Bushman, 2001; Anderson \& Bushman, 2018). Also, children acquire aggression from peer role models through observational learning (Bandura, 1978). However, little research has been conducted to examine the effects of weapon toys on aggressive behavior and related variables among six year olds in China. Thus, we attempt to test whether this effect will apply to six-year-old children and expect main effects of weapon toys and gender on outcome variables of aggressive cognition and behavior.

\section{Gender Effects on Aggression}

Extant research has found significant sex differences in trajectories of physical and social aggression (Katherine et al., 2008). Generally, males tend to show more aggression than females in Western countries (Björkqvist, 2018). In addition, gender socialization is regarded as a variable linked to the tendency to display aggressive behavior because boys are more prone to achieving manhood by maintaining a hierarchy of social superiority of masculinity ( $D^{\prime}$ Urso \& Pace, 2019). It is worth noting that boys are more likely to play with weapon toys than girls in the kindergarten, and that boys are more likely to solve interpersonal conflicts with physical aggression than girls. In view of this, there is a possible main effect of gender on aggression under the condition of weapon toys exposure.

\section{Aggressive Cognition as a Potential Mediator}


The first landmark research to discover the relationship between weapons and aggressive behavior (i.e., experiments on cue-elicited effects of weapons on aggressive behavior) showed that simply seeing a gun can increase aggression-called the "weapons effect" (Berkowitz \& LePage, 1967). Weapons are associated with aggression-related variables (Benjamin et al., 2018; Berkowitz, 1968). Previous research has also revealed that weapon pictures and weapon names automatically prime aggression (Anderson et al., 1998). Also, exposure to firearm/gun violence increases the possibility of subsequent violent behavior in areas like video games and movies (Bingenheimer et al., 2005; Chang \& Bushman, 2019; Dillon \& Bushman, 2017; Kleck, 2005; Mehta, 2005). Specifically, aggressive cartoons and toys cause more aggressive behavior than neutral cartoons and toys among preschool children (Sanson \& Muccio, 1993). The metaanalysis has indicated that exposure to violent video games leads to aggressive cognition, hostile feeling and aggressive behavior (Anderson et al., 2010). Conversely, other researchers did not find evidence that violent stimuli escalate aggression, and that the relationship between violent video games and aggression is minimal and even null (Ferguson \& Dyck, 2012; Ferguson \& Kilburn, 2010). The different conclusions concerning the media violence effect on aggression may be caused by the cultural variation (Ramirez et al., 2001). Given that China is a country that prohibits the use of firearms, children may have different perceptions of weapons relative to those in countries that allow the legitimate use of firearms. The culture of weapon use versus non-weapon use may lead children to form different cognition of weapon toys and aggressive behavior. In addition, previous study mainly focuses on the impact of weapon primes on adolescents or adults (Anderson et al., 1998). However, little is known about the weapon toy effects on Chinese children. To this end, it is necessary to evaluate the effect of weapon toys exposure on aggressive cognition and aggressive behavior among Chinese children.

\section{The Present Study}

Previous research literature experimentally shows automatic priming effects of weapon pictures and weapon words on aggression. However, there is a relative lack of weapon toy studies from Eastern cultures. The study aims to examine the short-term effects of brief weapon toys exposure versus non-weapon toys exposure on aggressive cognition and aggressive behavior in a group of Chinese children. We randomly assign boys and girls to either weapon or non-weapon toys condition and measure their aggressive cognition and behavior afterwards. The main research questions are as follows: (a) Does playing with weapon toys affect aggressive cognition and behavior among children? If so, are there any gender differences? (b) Are these weapon toy effects on aggressive behavior mediated by aggressive cognition? In order to find the answers to these questions, we employed a 2 (Toys) x 2 (Gender) experimental design to determine whether there are any differences in the levels of aggressive cognition and behavior for weapon toy players and non-weapon toy players to make a contribution to the field. Thus, we hypothesize that:

H1. Playing with weapon toys will increase aggressive cognition and aggressive behavior.

H2. Boys, but not girls, will show an increase in aggressive cognition and aggressive behavior in the context of weapon toys.

H3. Aggressive cognition will mediate the weapon toy effects on aggressive behavior.

\section{Methods \\ Participants}

A total of 104 six-year-old children ( $50 \%$ girls; $\left.M_{\text {age }}=6.19, S D=0.10\right)$ by class were randomly recruited from a Chinese kindergarten (group sampling). Specifically, our experimental staff came to organize the children to participate in the experiment with the help of their teachers. All the participants were righthanded and have no psychiatric disorders. Half of them were randomly assigned to play with weapon toys or non-weapon toys. There were 26 girls and 26 boys in the weapon toy condition, and 26 girls and 26 boys in the non-weapon toy condition. When children were playing with toys, they were playing in a group of 8 people each time [number of weapon group $=4$ (girls $=2$, boys $=2$ ), number of non-weapon group $=4$ ( girls $=2$, boys $=2$ )], constituting for 13 groups (girls $=52$, boys $=52$ ). Children in the playgroups might know each other from their classrooms. These children did not have previous history of serious aggression. They were not playing separately. Participants were not allowed to interact with other children when they played as a group. Their parents were not present in the hall when their children played with toys. No participants failed to complete the experiment.

\section{Design}

A 2 (Toys: weapon vs. non-weapon) x 2 (Gender: boys vs. girls) between-subjects experimental design was conducted. The independent variables were toys and gender. The dependent variables were aggressive cognition and aggressive behavior. Age was a covariate.

\section{Materials}

Sixty toys, including 30 weapon toys (e.g., Sword, Gun, Rifle, Axe) and 30 non-weapon toys (e.g., Doll, Thomas the Tank Engine, Helicopter), were used as toy materials/primes to trigger aggressive cognition and aggressive behavior. These weapon toys were more cartoonish because they look safer. We observed some Chinese children play with these weapon/non-weapon toys in their daily life, and thus decided to buy/select these particular toys as experimental primes to improve ecological validity. These children have more or less exposure to either weapon or non-weapon toys at their leisure.

\section{Aggressive Cognition}


In the current study, the Semantic Classification Task (SCT) or Picture Decision Task (PDT) was used to measure children's aggressive cognition. The SCT can be described a cognitive priming task that children classify target pictures/words (e.g., aggressive vs. non-aggressive) accurately and quickly by making reaction time (RT) responses (Bowers \& Turner, 2003). Children's reaction times (RTs) to weapon/aggressive pictures in the SCT were regarded as a marker of aggressive cognition. A total of 35 aggressive (e.g., guns, swords) and 35 non-aggressive pictures (e.g, flower, watermelon) were selected as target pictures. Aggressive/weapon pictures and non-aggressive/non-weapon pictures were used as cues. The SCT was performed on 15-inch laptops using E-prime psychology software. The SCT included practice session and formal session. In practice session, 5 weapon pictures and 5 non-weapon pictures were matched with 4 rounds, forming totally 40 trials. Namely, the number of aggressive pictures and non-aggressive pictures were equal, and the number of presented times that aggressive pictures vs. non-aggressive pictures were also equal. These pictures used in practice section would not appear in formal section. In practice session, experimenters sat aside and answered questions raised by participants. If the accuracy rates in practice session were lower than $85 \%$, participants should continue to practice until their accuracy rates were higher than $85 \%$. The purpose of practice session was to familiarize participants with the key pressing. In formal session, a total of 30 weapon pictures and 30 non-weapon pictures constituted for 60 trials. Specifically, a small fixation "+" emerged on the screen center for $200 \mathrm{~ms}$ to remind participants to prepare, then each picture was presented for $2000 \mathrm{~ms}$. Participants were required to press buttons as quickly and accurately as possible. If participants did not respond, the next trial would automatically appear. The RTs and accuracy rates were recorded. Experimenters read instructions aloud for each participant: " The experiment is to test the speed and accuracy of your response, you should judge whether the pictures are weapon/aggressive or non-weapon/non-aggressive by pressing ' 1 ' for weapon/aggressive pictures or pressing '2' for non-weapon/nonaggressive pictures. After your response, the next trial starts." Based on previous research (Bowers \& Turner, 2003), participants pressed buttons to identify the picture based on its semantic attributes (aggressive vs. non-aggressive). In lexical decision tasks that were often employed, longer RTs to weapons would mean less accessibility to aggressive cognition, shorter RTs to weapons would mean more accessibility.

\section{Aggressive Behavior}

A Competitive Reaction Time Task (CRTT) was used to measure aggressive behavior. Researchers have proved that CRTT is a valid measure for aggressive behavior in laboratory settings (Hyatt et al., 2019; Warburton \& Bushman, 2019). Each participant competed with a "virtual opponent" to see who responded first upon presentation of a tone, and participants who reacted more slowly were "punished" by a loud noise (e.g., Anderson \& Murphy, 2003). After each trial, the "loser" received a loud noise, the noise intensity was set by the "virtual opponent." The pattern of wins/losses and the noise intensity blasts were determined after the participant knew who won/lost the trial. The CRTT was a two-step paradigm according to previous researches (Anderson \& Murphy, 2003; Warburton \& Bushman, 2019). In this study, considering the kindergarten age children, we specifically reduced the volume of noise intensity to $70-100 \mathrm{~dB}$, which was less than the normal setting of noise intensity blasts, usually more than $100 \mathrm{~dB}$. In other words, the CRTT was specifically adapted for use with this younger children. Therefore, it was not able to be used exactly as described for adults.

In Step 1, there were 25 trials, in which the "virtual opponent" set the noise intensity (70-100 dB, weak-strong) for the participant on "loss" trials. Participants were given sample noise blasts of $70 \mathrm{~dB}$ and $100 \mathrm{~dB}$ before the Step 1, but they did not set noise intensity for the "virtual opponent" in Step 1. Each participant received the same series of 13 wins and 12 losses. Specifically, trial 1 was a "win" and the remaining 24 trials were divided into three blocks, with 8 trials in each block (4 wins vs. 4 losses). At the end of the Step 1, the experimenter reminded participants that they would set noise intensity for "virtual opponent" and would never receive noise in the next step. In Step 2, there were the same 25 trials as in Step 1, but the roles were reversed. The participant's noise intensity setting (70-100 dB) delivered to the "virtual opponent" represented aggressive behavior. If the participant was a winner, he/she could select one of the fourrank noise intensity $(70,80,90,100 \mathrm{~dB})$ by pressing corresponding button 1-4 (1-4 points) to punish the "virtual opponent". A "no" noise option (0 dB, no aggressive behavior) was available. The noise intensity (0-4 points) children set for "virtual opponent" in Step 2 of the CRTT was deemed as a marker of aggressive behavior. Noticeably, assistants did not instruct children to punish a "virtual opponent" by using the loud noises as the Milgram experiment (Abbott, 2016).

\section{Procedure}

The researchers' university Ethics Committee approved all procedures of the experiment. First, parents gave informed consent to their children's participation. They were told that the study concerned the effects of weapon toy play vs. non-weapon toy play on cognitive performances and noise intensity settings. Then the assistants explained the experimental procedure and reminded participants that they were allowed to stop the experiment as long as they felt uncomfortable. Second, half of the participants were randomly assigned to play with weapon toys or non-weapon toys for 20 minutes in a big quiet hall of the kindergarten. A big quiet hall meant a large auditorium of the kindergarten with spacious and bright activity space rather than a school hallway. Third, participants performed SCT and CRTT on 15-inch laptops. More precisely, children were measured aggressive cognition (making RT responses to 30 aggressive/weapon pictures) and aggressive behavior (setting noise intensity, 0-4 points), immediately after playing with toys in a group setting. Fourth, participants were debriefed on their aggressive feelings to assess suspicion because suspicion could be a problem in CRTT (e.g., Anderson et al., 1996). No participant expressed suspicion during the debriefing process. Finally, all participants were rewarded with nice stickers (i.e., incentives) from assistants after they completed the experiment. The experiment complied with the Declaration of Helsinki.

\section{Statistical Analysis}

We used SPSS 21.0 to conduct a 2 (Toys) x 2 (Gender) Analysis of Covariance (ANCOVA) to assess the effect of toys on aggressive cognition and behavior, with age controlled as a covariate. We also used PROCESS 3.0 to run mediation analysis to test the mediating effects of aggressive cognition. 


\section{Results \\ Descriptive Statistics}

The means, standard deviations, and cell sample sizes for each of the 4 conditions are presented in Table 1 (SCT measure) and Table 2 (CRTT measure). RTs to weapon/aggressive pictures represent levels of aggressive cognition. Accordingly, a "high aggressive cognition" means short RTs to aggressive pictures. Noise intensity settings represent levels of aggressive behavior. Accordingly, a "more aggressive behavior" means high noise intensity setting. We have conducted a G-Power 3.1 analysis to determine the prior sample size for this study. The priori required sample size computed was $N=52$. The power size is 0.95 . We believe the study sample size $(N=102)$ can meet this demand in regards to G-Power calculation. As we can see, RTs in a weapon toy condition are shorter than those in a non-weapon toy condition, and noise intensity settings in a weapon toy condition are higher than those in a non-weapon toy condition. Additionally, boys have shorter RTs and higher noise intensity settings than girls in a weapon toy condition. Thus, it seems that toys, gender, aggressive cognition and aggressive behavior have a certain relationship.

Table 1

Descriptive statistics for aggressive cognition in the SCT (ms)

\begin{tabular}{|llllc|}
\hline Gender & Weapon toys & \multicolumn{3}{c|}{ Non-weapon toys } \\
& $M \pm S D$ & $M \pm S D$ & $N$ \\
\hline Boys & $1033.68 \pm 86.72$ & 26 & $1327.19 \pm 80.22$ & 26 \\
Girls & $1212.88 \pm 249.14$ & 26 & $1335.44 \pm 293.71$ & 26 \\
Total & $1123.28 \pm 205.67$ & 52 & $1331.32 \pm 213.21$ & 52 \\
\hline Note. SCT, semantic classification task (reaction times to weapon pictures). ms, milliseconds. \\
\hline
\end{tabular}

Table 2

Descriptive statistics for aggressive behavior in the CRTT (dB)

\begin{tabular}{|llllc|}
\hline Gender & Weapon toys & \multicolumn{3}{c|}{ Non-weapon toys } \\
& $M \pm S D$ & $N$ & $M \pm S D$ & $N$ \\
\hline Boys & $3.59 \pm 0.42$ & 26 & $1.54 \pm 0.49$ & 26 \\
Girls & $2.74 \pm 0.95$ & 26 & $2.49 \pm 1.05$ & 26 \\
Total & $3.16 \pm 0.84$ & 52 & $2.02 \pm 0.94$ & 52 \\
\hline Note. CRTT, competitive reaction time task (noise intensity setting). dB, decibel. \\
\hline
\end{tabular}

\section{Aggressive Cognition}

To test $\mathrm{H} 1$ and $\mathrm{H} 2$, we conducted a 2 (toys) x 2 (gender) Analysis of Covariance (ANCOVA) to examine the effects of toys on aggressive cognition (RTs to weapon pictures), with age included as a covariate. The main effect of toys on aggressive cognition was significant. Playing with weapon toys primed higher aggressive cognition (shorter RTs to aggressive pictures) than playing with non-weapon toys $[F(1,99)=27.46, p<0.001, d=1.04 ; M=1123.16(S E=28.09)<$ $M=1331.44$ ( $S E=28.09$ )]. The main effect of gender on aggressive cognition was significant. Boys primed higher aggressive cognition (shorter RTs to aggressive pictures) than girls $[F(1,99)=5.57, p=0.02, d=0.47 ; M=1180.15(S E=28.17)<M=1274.45(S E=28.17)]$. In addition, the toys $\times$ gender interaction on aggressive cognition was significant $\left[F(1,99)=4.63, p=0.03, \eta_{p}{ }^{2}=0.04\right]$. A simple effect analysis indicated that boys in a weapon toy condition displayed significant higher aggressive cognition than those in a non-weapon toy condition $\left[F(1,99)=27.35, p<0.001, \eta_{p}^{2}=0.21 ; M=1033.31(S E=39.81)<\right.$ $M=1326.99(S E=39.73)$ ]. Likewise, girls in a weapon toy condition displayed significant higher aggressive cognition than those in a non-weapon toy condition $\left[F(1,99)=4.78, p=0.03, \eta_{p}^{2}=0.04 ; M=1213.01(S E=39.71)<M=1335.89(S E=39.87)\right](F i g .1)$.

\section{Aggressive Behavior}

To test $\mathrm{H} 1$ and $\mathrm{H} 2$, we conducted a 2 (Toys) x 2 (Gender) ANCOVA to examine the effects of toys on aggressive behavior (noise intensity setting), with age included as a covariate. The main effect of toys on aggressive behavior was significant. Playing with weapon toys led to more aggressive behavior (higher noise intensity) than playing with non-weapon toys $[F(1,99)=55.98, p<0.001, d=1.48 ; M=3.17(S E=0.11)>M=2.02(S E=0.11)]$. However, the main effect of gender on aggressive behavior was not significant $[F(1,99)=0.08, p=0.78, d=0.06]$. In addition, the toys $\mathrm{x}$ gender interaction on aggressive behavior was significant $\left[F(1,99)=34.06, p<0.001, \eta_{p}^{2}=0.25\right]$. A simple effect analysis indicated that boys in a weapon toy condition displayed significant more 
aggressive behavior than those in a non-weapon toy condition $\left[F(1,99)=88.77, p<0.001, \eta_{p}^{2}=0.47 ; M=3.59(S E=0.15)>M=1.55(S E=0.15)\right]$. However, no significant group differences in aggressive behavior were found among girls $\left[F(1,99)=1.37, p=0.25, \eta_{p}^{2}=0.01\right]($ Fig. 2).

\section{Mediation Analysis}

To test $\mathrm{H} 3$, we further run a mediation analysis on the effects of aggressive cognition on aggressive behavior (standardized coefficients) after weapon toys exposure, with gender controlled as a covariate because of the significant toys $x$ gender interaction. In addition, the above ANCOVA results also showed that playing with weapon toys increased aggressive cognition and behavior simultaneously. The methods for single-mediator models and bootstrapping (number of bootstrap samples for bias-corrected bootstrap $95 \%$ confidence intervals $=5,000$ ) were used to assess the magnitude of the indirect effect. There was a significant mediation if the $95 \% \mathrm{Cl}$ for indirect effect did not include zero (Hayes \& Preacher, 2014), and vice versa.

As shown in Fig. 3, the direct effect of weapon toys on aggressive behavior was significant [c' $=0.26 ; S E=0.11 ; 95 \% \mathrm{Cl}$ : $(0.04 ; 0.49)$ ]. Moreover, weapon toys significantly predicted high aggressive cognition (short RTs to weapon pictures) [a $=-0.65 ; S E=0.06 ; 95 \% \mathrm{Cl}$ : $(-0.77 ;-0.52)]$, and high aggressive cognition (short RTs to weapon pictures) significantly predicted high aggressive behavior (high noise intensity) $[\mathrm{b}=-0.43 ; S E=0.13 ; 95 \% \mathrm{Cl}$ : $(-0.68 ;-0.18)]$. Thus, aggressive cognition partially mediated the effect of weapon toys on aggressive behavior [ab $=0.28 ; S E=0.08 ; 95 \% \mathrm{Cl}$ : $(0.09 ; 0.41)$ ]. The total effect of weapon toys on aggressive behavior was also significant $(c=0.54, S E=0.08,95 \% \mathrm{Cl}[0.38,0.71])$. The direct effect $\left(c^{\prime}=0.26\right)$ and the mediating effect $(a b=$ 0.28 ) accounted for $48 \%$ and $52 \%$ of the total effect $(c=0.54)$, respectively. In the current research, both aggressive cognition and aggressive behavior increased for children who played with weapon toys. As expected, more weapon toys predicted higher aggressive cognition (shorter RTs to weapon pictures), and higher aggressive cognition predicted higher aggressive behavior (higher noise intensity).

\section{Discussion}

\section{The Implications of The Findings}

The study may have potential broad implications for children to play with weapon toys and prevent aggression. First, children playing with weapon toys (versus non-weapon toys) display an increase in aggressive cognition and behavior, suggesting that guardians may reduce the number of times that 6-yearolds play with weapon toys. Second, boys playing with weapon toys display more aggressive cognition and aggressive behavior than those playing with nonweapon toys, suggesting that boys (versus girls) may receive more attention in real-life settings when playing with weapon toys. However, we may pay particular attention to avoid reinforcing gender stereotypes when discussing this issue. Finally, given that aggressive cognition partially mediated the weapon toy effects on aggressive behavior, educators may alleviate aggressive cognition to reduce children's subsequent aggressive behavior. However, the fact that primed accessibility of aggressive cognition (or feelings of aggression) increases aggressive behavior does not necessarily mean that aggressive cognition always lead to violent acts due to the short-term weapon toy effects in the cross-sectional study.

It is worth noting that many Chinese children are also playing with weapon toys in daily life when they are not in the kindergarten. However, our experiment can not provide direct and definite answers to children's aggressive cognition and behavior after playing with weapon toys when they are not in the kindergarten. Because the experimental study was conducted in the Big Hall of the kindergarten, and this experimental situation is different from the natural settings to some extent. This is just like Bandura's experiment of aggressive models with BoBo dolls (Bandura et al., 1961). In other words, the present study cannot answer the question whether the findings in the context of kindergarten can be generalized to other places or not. Based on the findings from the present study, we can only postulate that children who play with weapon toys inside the kindergarten will be more likely to display an increase in aggressive behavior and cognition in comparison with those who play with non-weapon toys. Accordingly, we should challenge the use of these weapon toys for children as a result of our findings.

Consistent with Hypothesis 1, we found that playing with weapon toys lead to more aggressive cognition and aggressive behavior than playing with nonweapon toys. The results also supported previous research that weapons automatically primed aggression (Bartholow et al., 2005; Wolff, 1976) and aligned with the weapon effect on aggression (Berkowitz \& LePage, 1967). According to the GAM and extant literature (Anderson \& Bushman, 2018; Khoury, 2012), situational factors and personal factors have interactive effects on aggressive cognition and behavior. Why does playing with weapon toys increase aggressive cognition and aggressive behavior among children? On the one hand, perhaps weapon toys may decrease the level of empathy in children and increase hostile attribution bias (Gao et al., 2017; Holden, 2005; Strasburger \& Wilson, 2002). On the other hand, playing with weapon toys may increase children's cognitive biases towards weapon pictures and raise aggressive behaviors. The possible reason is that participants may associate weapon cues with aggression-related constructs in cognitive-associated networks (Berkowitz, 2017). Meanwhile, children consume cognitive resources to identify aggressive versus non-aggressive pictures due to their primed rehearsed knowledge structure (Bushman, 1998). In addition, prior researchers found that weapon pictures and weapon names automatically trigger aggression (Anderson et al., 1998). In view of this, parents and teachers may prevent children from playing with weapons toys or reduce the chance of playing with weapons toys, so as to avoid potential aggressive cognition and behavior.

With regard to Hypothesis 2, we found that boys in a weapon toy condition demonstrated more aggressive cognition and aggressive behavior than those in a non-weapon toy condition, whereas girls in a weapon toy condition only demonstrated more aggressive cognition (but not aggressive behavior) than those in a non-weapon toy condition. The finding was consistent with previous studies that boys behaved aggressively in comparison with girls (Boutwell et al., 2011; Card et al., 2008; Rose \& Rudolph, 2006; Smith \& Waterman, 2005). Why are gender differences emerged? Why do girls show increased aggressive cognition instead of aggressive behavior in a weapon toy condition? First, perhaps girls are better at restraining their aggressive cognition in order to inhibit aggressive behavior than boys in the context of weapon toys, whereas boys are more prone to take violent action in order to vent their anger than girls (Engelhardt et al., 2011; Giumetti \& Markey, 2007; Yao et al., 2019). Second, perhaps girls are better at showing an increase in empathy than boys to decrease aggression after

Page $6 / 12$ 
exposure to violence (Gao et al., 2017; Krahé \& Möller, 2010). The finding indicates that parents and teachers should manage aggressive behavior for boys and girls in different ways when they are involved in weapon toy games. Third, we assume that possible socialization effects are appropriate to explain the gender differences in weapons effects (Eron, 1992; Gallina \& Fass, 2014). Gender-differentiated parenting greatly affects children's socialization from traditional Chinese cultures (Ngai et al., 2018). For example, prior research has demonstrated that parents are more likely to adopt strict strategies to discipline males even when they exhibit the same misconducts as females (Cui \& Lan, 2020; Xing et al., 2019). Previous research has shown that direct aggression (e.g., physical) is more common in males than females across cultures, and aggression occurs during early childhood because of sexual selection and social role (Archer, 2004). In addition, the fact that females are less aggressive than males across cultures compels the serious consideration of a social learning basis, such as gender socialization, parents' gender-role stereotypes (Endendijk et al., 2016). Thus socialization effects may cause the gender differences in aggression when children are playing with weapon toys.

Consistent with Hypothesis 3, we found that the weapon toy effect on aggressive behavior is partially mediated by increased aggressive cognition. In addition, the finding supports the GAM and extant related-researches that media violence increases aggressive behavior through aggressive cognition and aggressive thoughts (e.g., Anderson \& Bushman, 2001; Anderson \& Bushman, 2018; Carnagey \& Anderson, 2005). The explanation is that children's cognitive networks of aggression are likely to be primed by playing with weapons toys, which consequently leads to follow-up aggressive behavior. Therefore, we can help adults be aware of weapons effects and limit the access that young children have of toy weapons.

\section{Strengths, Limitations, and Implications}

Although prior researches indicated that media violence begets aggression in Western nations, little researches touched on the weapon toy effects on children in Eastern nations. Thus, our study may contribute to the existing literature. First, we used an experimental design to test the salience of the weapons effect on children in an Eastern sample, and it illustrates a relation between weapon toys manipulation and aggression-related variables. A major strength of the study was that the independent variable of weapon toys was a true experimental variable. Second, we used a non-Western sample of children in Eastern cultures, and the ability to examine gender differences in weapon toy effects. The cultural facet and the fact that it was performed in China could be of value to this field. This could also be regarded as a strong point in our study. Third, the potential strength of this study was that it gave us an insight into the weapon toy effects on aggressive behavior through increased aggressive cognition.

Of course, given that previous researchers have tested the weapon effects using Western samples (Anderson et al., 1998; Berkowitz \& LePage, 1967), thus the finding using non-Western samples may not provide a novelty in this field, and some limitations should be noted. First, the sample is relatively small and homogeneous. As such, generalized validity will be questioned when applying conclusions to other age groups, and we should include a discussion of broader societal issues to elevate its potential impact in future. Second, children's interests in these weapon toys may not be the same, future research should allow the them to choose toys which are rated in terms of interest, pleasure, excitement, difficulty, violence, and so on. In other words, gender differences could be explained by acceptance of traditional gender roles. Because boys may be more interested in playing with some types of weapon toys than girls, and therefore selecting weapon toys also attracting girls is necessary. Third, the lab-based aggression paradigm (white noise blasts) may not be generalizable to physical aggression and relational aggression of children in real-world settings. Actually, the label of aggression is often associated with acts of physical aggression (e.g., pushing, hitting) or bullying (e.g., relational aggression, threats). While the noise intensity task is interesting and is an ethical way of measuring "aggression" in the laboratory, it really only infers aggressive behavior. Thus, it may be an overstatement to suggest that playing with weapon toys "leads to" realistic aggression. In this regard, more cautions are needed when interpreting the findings related to "aggressive behavior" in the noise intensity task. Because few studies have proved that this noise intensity task is related to observed or self-reported physical or relational aggression in the real world. We may specifically address this potential lack of ecological validity between lab-based aggression and realistic aggression in future directions. Fourth, although children in the playgroups might know each other from their classrooms and did not have previous history of serious aggression, we did not specifically test whether the combination of children with previous history together (whether good or bad) had contributed to the types of play exhibited. Finally, this study lacks of reporting from an older person like kindergarten teachers for the validity of research results. Future work should consider adopting the reporting from older persons to improve the validity.

\section{Conclusions}

The current study explored the relationships between exposure to weapon toys and aggressive cognition and behavior among children in a specific region in China. Through a randomized controlled trial it was found that playing with weapon toys can significantly increase aggressive cognition and behavior among Chinese children. Boys are more likely than girls to exhibit aggressive behavior in the context of weapon toys, though girls do exhibit aggressive cognition. Aggressive cognition partially mediates the effects of weapon toys on aggressive behavior. Boys should be considered as the target group for aggression intervention and reduction. The importance and relevance of the study reported lies in that educators should take the weapon toys away given the results and decrease the levels of aggressive cognition to reduce children's aggressive behavior.

\section{Declarations}

\section{Ethics approval and consent to participate}

The study involving human participants was reviewed and approved by Southwest University in China. Parents gave informed consents to their children's participation in the study after a detailed explanation of the study protocol. 


\section{Consent for publication}

We give consents for publication of this article.

\section{Availability of data and materials}

The datasets generated for this study are available from this URL: https://osf.io/vfstz/

\section{Competing interests}

There are no competing interests in regards to this article.

\section{Funding}

The current study was supported by the National Social Science Foundation of China (17CSH006) and the Central University' s Fundamental Grant (SWU2009201).

\section{Authors' contributions}

QZ conceived and designed the experiments. QZ performed the experiments. QZ performed the statistical analyses and critically revised the manuscript. QZ wrote the paper. The author read and approved the final draft of the manuscript.

\section{Acknowledgements}

We gratefully acknowledge the support of the kindergartners and their parents who participated in this project. We thank graduate student Yi Cao and Dr. YanGu Pan for their thoughtful feedback on the earlier draft of this manuscript. Special thanks should also be given to Ms. JingJin Tian, the head of Dawn Innovation Kindergarten, for her charismatic organization and arrangement. Moreover, we are grateful to the editor and the reviewers for their guidance and constructive comments concerning the revision of the manuscript.

\section{References}

1. Abbott, A. (2016). Modern Milgram experiment sheds light on power of authority. Nature, 530(7591), 394. https://doi.org/10.1038/nature.2016.19408

2. Anderson, C. A., Anderson, K. B., \& Deuser, W. E. (1996). Examining an affective aggression framework weapon and temperature effects on aggressive thoughts, affect, and attitudes. Personality and Social Psychology Bulletin, 22(4), 366-376. https://doi.org/10.1177/0146167296224004

3. Anderson, C. A., Benjamin, A. J., \& Bartholow, B. D. (1998). Does the gun pull the trigger? Automatic priming effects of weapon pictures and weapon names. Psychological Science, 9(4), 308-314. https://doi.org/10.1111/1467-9280.00061

4. Anderson, C. A., \& Bushman, B. J. (2001). Effects of violent video games on aggressive behavior, aggressive cognition, aggressive affect, physiological arousal, and prosocial behavior: A meta-analytic review of the scientific literature. Psychological Science, 12(5), 353-359. https://doi.org/10.1111/14679280.00366

5. Anderson, C. A., \& Bushman, B. J. (2002). Human aggression. Annual Review of Psychology, 53(1), 2751. https://doi.org/10.1146/annurev.psych.53.100901.135231

6. Anderson, C. A., \& Bushman, B. J. (2018). Media violence and the General Aggression Model. Journal of Social Issues, 74(2), 386413. https://doi.org/10.1111/josi.12275

7. Anderson, C. A., \& Murphy, C. R. (2003). Violent video games and aggressive behavior in young women. Aggressive Behavior, 29(5), 423429. https://doi.org/10.1002/ab.10042

8. Anderson, C. A., Shibuya, A., Ihori, N., Swing, E. L., Bushman, B. J., Sakamoto, A., \& Saleem, M. (2010). Violent video game effects on aggression, empathy, and prosocial behavior in Eastern and Western countries: A meta-analytic review. Psychological Bulletin, 136(2), 151-173.

https://doi.org/10.1037/a0018251

9. Archer, J. (2004). Sex differences in aggression in real-world settings: A meta-analytic review. Review of General Psychology, 8(4), $291-322$. https://doi.org/10.1037/1089-2680.8.4.291

10. Bandura, A. (1999). Social cognitive theory: An agentic perspective. Asian Journal of Social Psychology, 2(1), 21-41. https://doi.org/10.1111/1467839X.00024

11. Bandura, A. (1978). Social learning theory of aggression. Journal of Communication, 28(3), 12-29. https://doi.org/10.1111/j.1460-2466.1978.tb01621.x

12. Bandura, A., Ross, D., \& Ross, S. A. (1961). Transmission of aggression through imitation of aggressive models. Journal of Abnormal and Social Psychology, 63(3), 575-582. https://doi.org/10.1037/h0045925

13. Bartholow, B. D., Anderson, C. A., Carnagey, N. L., \& Benjamin, A. J. (2005). Interactive effects of life experience and situational cues on aggression: The weapons priming effect in hunters and nonhunters. Journal of Experimental Social Psychology, 41(1), 48-60. https://doi.org/10.1016/j.jesp.2004.05.005 
14. Berkowitz, L. (2017). On automatic as well as controlled psychological processes in aggression: From the cognitive neoassociation perspective. In $P$. Sturmey (Ed.), The Wiley handbook of violence and aggression. John Wiley \& Sons, Ltd. https://doi.org/10.1002/9781119057574.whbva038

15. Berkowitz, L. (1968). Impulse, aggression and the gun. Psychology Today, 2(9), 19-22.

16. Berkowitz, L., \& Lepage, A. (1967). Weapons as aggression-eliciting stimuli. Journal of Personality and Social Psychology, 7(2), $202-207$. https://doi.org/10.1037/h0025008

17. Benjamin, A. J., Kepes, S., \& Bushman, B. J. (2018). Effects of weapons on aggressive thoughts, angry feelings, hostile appraisals, and aggressive behavior: A meta-analytic review of the weapons effect literature. Personality and Social Psychology Review, 22(4), 347-377. https://doi.org/10.1177/1088868317725419

18. Bingenheimer, J. B., Brennan, R. T., \& Earls, F. J. (2005). Sociology: Firearm violence exposure and serious violent behavior. Science, 308(5726), 13231326. https://doi.org/10.1126/science. 1110096

19. Björkqvist, K. (2018). Gender differences in aggression. Current Opinion in Psychology, 19(2), 39-42. https://doi.org/10.1016/j.copsyc.2017.03.030

20. Boutwell, B. B., Franklin, C. A., Barnes, J. C., \& Beaver, K. M. (2011). Physical punishment and childhood aggression: The role of gender and geneenvironment interplay. Aggressive Behavior, 37(6), 559-568. https://doi.org/10.1002/ab.20409

21. Bowers, J. S., \& Turner, E. L. (2003). In search of perceptual priming in a semantic classification task. Journal of Experimental Psychology: Learning, Memory, and Cognition, 29(6), 1248-1255. https://doi.org/10.1037/0278-7393.29.6.1248

22. Bushman, B. J. (1998). Priming effects of media violence on the accessibility of aggressive constructs in memory. Personality and Social Psychology Bullı 545. https://doi.org/10.1177/0146167298245009

23. Bushman, B. J., \& Huesmann, L. R. (2010). Aggression. In Fiske ST, Gilbert, D. T., Lindzey, G. (Eds.), Handbook of social psychology (5th ed., pp. 833-863). New York: John Wiley \& Sons.

24. Card, N. A., Stucky, B. D., Sawalani, G. M., \& Little, T. D. (2008). Direct and indirect aggression during childhood and adolescence: A meta-analytic review of gender differences, inter-correlations, and relations to maladjustment. Child Development, 79(5), 1185-1229. https://doi.org/10.1111/j.14678624.2008.01184.x

25. Carnagey, N. L., \& Anderson, C. A. (2005). The effects of reward and punishment in violent video games on aggressive affect, cognition, and behavior. Psychological Science, 16(11), 882-889. https://doi.org/10.1111/j.1467-9280.2005.01632.x

26. Chang, J. H., \& Bushman, B. J. (2019). Effect of exposure to gun violence in video games on children's dangerous behavior with real guns: A randomized clinical trial. JAMA, 2(5), e194319. https://doi.org/10.1001/jamanetworkopen.2019.4319

27. Cui, G. Y., \& Lan, X. Y. (2020). The Associations of parental harsh discipline, adolescents' gender, and grit profiles with aggressive behavior among Chinese early adolescents. Frontiers in Psychology, 11(3), 323-337. https://doi.org/10.3389/fpsyg.2020.00323

28. Dillon, K. P., \& Bushman, B. J. (2017). Effects of exposure to gun violence in movies on children's interest in real guns. JAMA Pediatrics, 171(11),10571062. https://doi.org/10.1001/jamapediatrics.2017.2229

29. D'Urso, G., \& Pace, U. (2019). Homophobic bullying among adolescents: The role of insecure-dismissing attachment and peer support. Journal of LGBT Youth, 16(2), 173-191. https://doi.org/10.1080/19361653.2018.1552225

30. Endendijk, J. J., Groeneveld, M. G., Van, P. L. D., VanBerkel, S. R., Hallers-Haalboom, E. T., Bakermans-Kranenburg, M. J., et al. (2016). Gender differences in child aggression: Relations with gender-differentiated parenting and parents' gender-role stereotypes. Child Development, 88(1), 299-316. https://doi.org/10.1111/cdev.12589

31. Engelhardt, C. R., Bartholow, B. D., \& Saults, J. S. (2011). Violent and nonviolent video games differentially affect physical aggression for individuals high vs. low in dispositional anger. Aggressive Behavior, 37(6), 539-546. https://doi.org/10.1002/ab.20411

32. Eron, L. D. (1992). Gender differences in violence: Biology and/or socialization?. In Bjorkqvist K, Niemela P. Of mice and women: Aspects of female aggression (PP. 89-97). San Diego, CA: Academic Press. https://doi.org/10.1016/B978-0-12-102590-8.50013-1

33. Ferguson, C. J., \& Dyck, D. (2012). Paradigm change in aggression research: The time has come to retire the general aggression model. Aggression and Violent Behavior, 17(3), 220-228. https://doi.org/10.1016/j.avb.2012.02.007

34. Ferguson, C. J., \& Kilburn, J. (2010). Much ado about nothing: The misestimation and overinterpretation of violent video game effects in Eastern and Western nations: Comment on Anderson et al. (2010). Psychological Bulletin, 136(2), 174-178. https://doi.org/10.1037/a0018566

35. Gallina, M., \& Fass, W. (2014). The weapons effect in college females. Violence and Gender, 1(4), 165-169. https://doi.org/10.1089/vio.2014.0020

36. Gao, X. M., Weng, L., Zhou, Y., \& Yu, H. (2017). The Influence of empathy and morality of violent video game characters on gamers' aggression. Frontiers in Psychology, 8(11), 1863-1870. https://doi.org/10.3389/fpsyg.2017.01863

37. Geen, R. G. (2001). Human aggression (2nd ed.). Taylor \& Francis.

38. Giumetti, G. W., \& Markey, P. M. (2007). Violent video games and anger as predictors of aggression. Journal of Research in Personality, 41(6), 1234-1243. https://doi.org/10.1016/j.jrp.2007.02.005

39. Hayes, A. F., \& Preacher, K. J. (2014). Statistical mediation analysis with a multicategorical independent variable. British Journal of Mathematical and Statistical Psychology, 67(3), 451-470. https://doi.org/10.1111/bmsp.12028

40. Holden, C. (2005). Controversial study suggests seeing gun violence promotes it. Science, 308(5726), 12391240. https://doi.org/10.1126/science.308.5726.1239a

41. Hyatt, C. S., Chester, D. S., Zeichner, A., \& Miller, J. D. (2019). Analytic flexibility in laboratory aggression paradigms: Relations with personality traits vary (slightly) by operationalization of aggression. Aggressive Behavior, 45(4), 377-388. https://doi.org/10.1002/ab.21830

Page $9 / 12$ 
42. Katherine, K. J., Foshee, V. A., Ennett, S. T., \& Suchindran, C. (2008). The development of aggression during adolescence: Sex differences in trajectories of physical and social aggression among youth in rural areas. Journal of Abnormal Child Psychology, 36(8), 1227-1236. https://doi.org/10.1007/s10802008-9245-5

43. Khoury, K. M. (2012). Perpetration of aggressive behaviors against peers and teachers as predicted by student and contextual factors. Aggressive Behavior, 38(4), 253-262. https://doi.org/10.1002/ab.21424

44. Kleck, G. D. (2005). Firearms, violence, and self-protection. Science, 309(5741), 1675-1676. https://doi.org/10.1126/science.309.5741.1675

45. Krahé, B., \& Möller, I. (2010). Longitudinal effects of media violence on aggression and empathy among German adolescents. Journal of Applied Developmental Psychology, 31(5), 401-409. https://doi.org/10.1016/j.appdev.2010.07.003

46. Mehta, N. G. (2005). The sight of violence and violent action. Science, 309(5741), 1676-1677. https://doi.org/10.1126/science.309.5741.1676

47. Ngai, S. S. Y., Xie, L., Ng, Y. H., \& Ngai, H. L. (2018). The effects of parenting behavior on prosocial behavior of Chinese adolescents in Hong Kong. Children and Youth Services Review, 87(1), 154-162. https://doi.org/10.1016/j.childyouth.2018.02.030

48. Ramirez, J. M., Andreu, J. M., \& Fujihara, T. (2001). Cultural and sex differences in aggression: A comparison between Japanese and Spanish students using two different inventories. Aggressive Behavior, 27(4), 313-322. https://doi.org/10.1002/ab.1014

49. Rose, A., \& Rudolph, K. D. (2006). A review of sex differences in peer relationship processes: Potential trade-offs for the emotional and behavioral development of girls and boys. Psychological Bulletin, 132(1), 98-131. https://doi.org/10.1037/0033-2909.132.1.98

50. Rosenblum, B. (2007). Toy weapon with a periscope suitable for allowing a user to view obstructed objects through the periscope. United States Patent 7349153.

51. Sanson, A., \& Muccio, C. (1993). The influence of aggressive and neutral cartoons and toys on the behaviour of preschool children. Australian Psychologist, 28(2), 93-99. https://doi.org/10.1080/00050069308258882

52. Smith, P., \& Waterman, M. (2005). Sex differences in processing aggression words using the emotional Stroop task. Aggressive Behavior, 31(3), 271282. https://doi.org/10.1002/ab.20071

53. Strasburger, V. C., \& Wilson, B. J. (2002). Children, adolescents, and the media. Thousand Oaks, CA: Sage.

54. Surette, R., \& Maze, A. (2015). Video game play and copycat crime: An exploratory analysis of an inmate population. Psychology of Popular Media Culture, 4(4), 360-374. https://doi.org/10.1037/ppm0000050

55. Warburton, W. A., \& Bushman, B. J. (2019). The competitive reaction time task: The development and scientific utility of a flexible laboratory aggression paradigm. Aggressive Behavior, 45(4), 389-396. https://doi.org/10.1002/ab.21829

56. Wolff, C. M. (1976). The effects of aggressive toys on aggressive behavior in children. Dissertation Graduate Student Theses, Doctor of Philosophy (PhD) Dissertations, \& Professional Papers, The University of Montana. https://scholarworks.umt.edu/etd/9957

57. Xing, X., Zhang, L., Wei, Y., \& Wang, Z. (2019). Parental harsh discipline and preschooler's inhibitory control in China: Bidirectional relations and gender differences. Journal of Interpersonal Violence, Jun 13 (online publication). https://doi.org/10.1177/0886260519854558

58. Yao, M. Y., Zhou, Y. H., Li, J. Y., \& Gao, X. M. (2019). Violent video games exposure and aggression: The role of moral disengagement, anger, hostility, and disinhibition. Aggressive Behavior, 45(6), 662-670. https://doi.org/10.1002/ab.21860

\section{Figures}




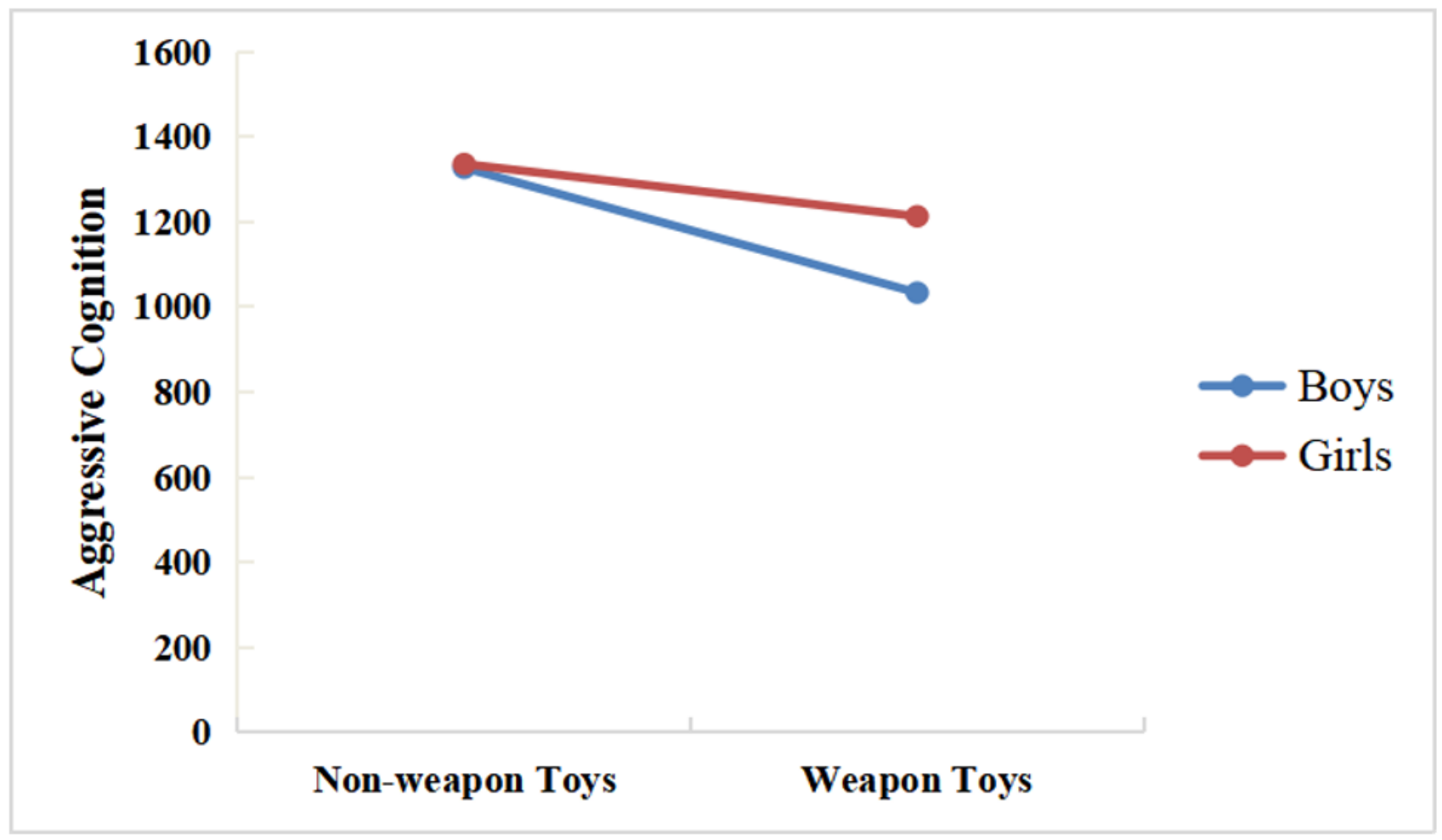

Figure 1

Interaction between toys and gender on aggressive cognition.

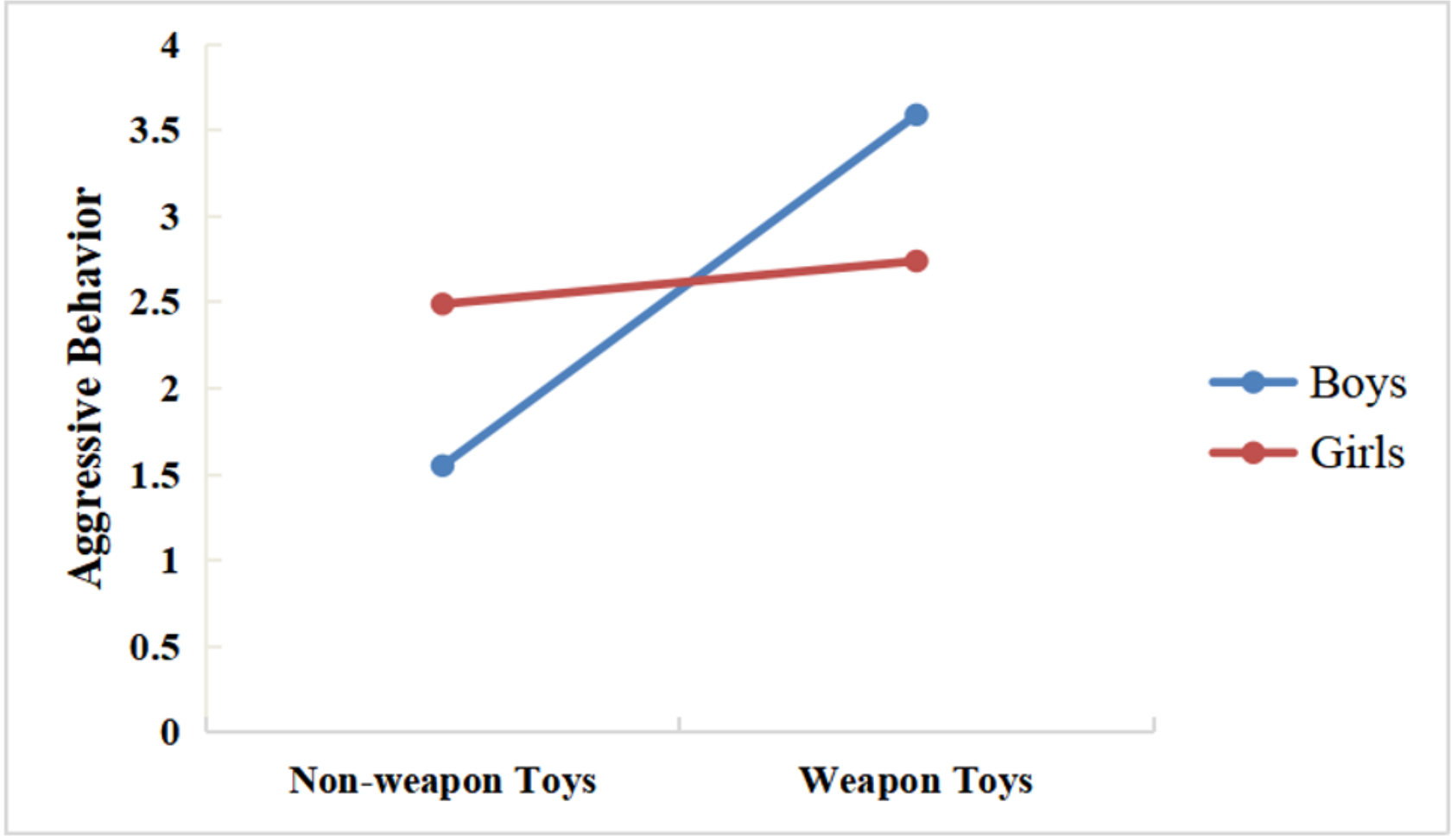

Figure 2

Interaction between toys and gender on aggressive behavior. 


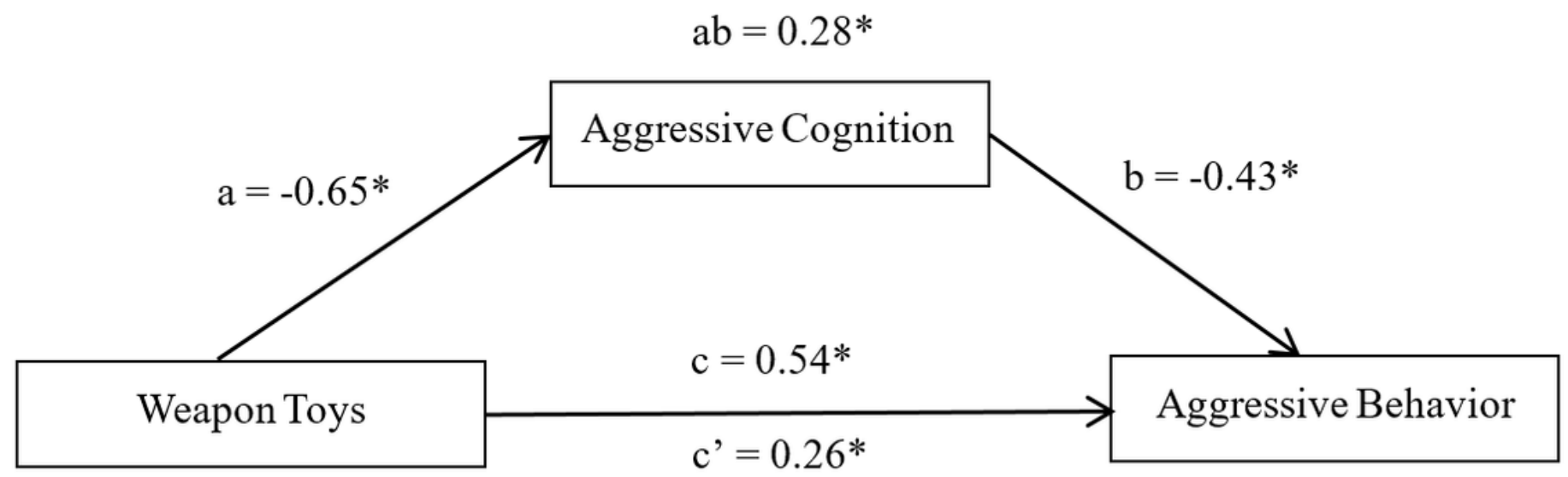

Figure 3

Mediation model of weapon toys on aggressive behavior through aggressive cognition. Note: $1=$ weapon, 0 = non-weapon; standardized path coefficients; Solid lines represent significant paths. *Statistically significant. 